\title{
COB-2019-2216
}

\section{AN INVERSE PROBLEM VIA CROSS-ENTROPY METHOD FOR CALIBRATION OF A DRILL STRING TORSIONAL DYNAMIC MODEL}

\section{Eber Dantas}

Americo Cunha Jr

Francisco José da Cunha Pires Soeiro

Universidade do Estado do Rio de Janeiro

eber.paiva@uerj.br, americo@ime.uerj.br, soeiro@uerj.br

Bruno Cesar Cayres

Centro Federal de Educação Tecnológica Celso Suckow da Fonseca - RJ

bruno.cayres@cefet-rj.br

\section{Hans Ingo Weber}

Pontifícia Universidade Católica do Rio de Janeiro

hans@puc-rio.br

Abstract. Model selection and parameter identification are challenging tasks in drill string dynamics due to the high degree of nonlinearity that abound the diverse and complex mechanisms involved. This work explores the application of a stochastic metaheuristic procedure for parameter identification over the torsional mode of drill string vibration. A proposed model is calibrated with data from a validated experimental set-up, adjusting stiffness, damping and friction parameters. The resulting simulations display a reasonable fit with almost ideal correlation coefficients even in the presence of stick-slip. The optimization strategy is compared with an Genetic Algorithm, revealing significantly greater efficiency and showcasing how the cross-entropy method may be a viable tool in the demanding context of drill string modeling.

Keywords: drill string dynamics, inverse problem, nonlinear dynamics, parameter identification, cross-entropy method

\section{INTRODUCTION}

The equipment used in oil prospecting operations, called drill string, is a long, slender structure under rotation, connected by drill-pipes and auxiliary apparatus. Diverse mechanisms influence in its performance: nonlinear interaction of multiple vibration modes, friction and shock effects, internal and external non-Newtonian flow, hysteretic interaction on the rock contact, etc (Wiercigroch et al., 2018; Spanos et al., 2003). The complexity of its dynamics warrants further works on the modeling approach and the use of numerical procedures that are efficient on the identification of its underlying values (Cunha Jr et al., 2015; Liao et al., 2018; Aguiar, 2010). For analysis over any dynamical system, calibration is fundamental. The correct assessment of parameter values is necessary to judge the capacity of a model in simulating phenomena under realistic measures; it is, in fact, a first step in a validation process concerning its properties and feasibility of its predictions (Pereira et al., 2018; Real et al., 2018). It is also a prerequisite in many uncertainty quantification techniques, where sensitivity analysis and the incorporation of variabilities may be developed over the best combination of inputs that reproduce a desired system response (Cunha Jr, 2015; Real et al., 2019).

The Cross-Entropy (CE) method is a stochastic metaheuristic global search algorithm that originated on the topics of rare-event simulation (Rubinstein, 1997; Rubinstein and Kroese, 2004) and combinatorial optimization (Eshragh et al., 2009), showing promising results and adaptability also to continuous optimization (Rubinstein, 1999; Rubinstein et al., 2006). However, in the subject of parameter identification, the method has not achieved common use as other metaheuristic algorithms. The easy implementation and conceptual insight of the procedure are very motivating reasons to check how it compares to similar optimization strategies that became classic in this area.

This work is part of a current project investigating the usefulness of the CE method in engineering applications (Dantas et al., 2019; Dantas, 2019), which is used here for calibration in the context of drill string dynamics. The global search capabilities and efficiency of the method are verified through the solution of an 
inverse problem that seeks to identify parameter values on the torsional dynamics of a validated experimental set-up (Cayres, 2018; Cayres et al., 2019). For the torsional mode vibration, a common subject is the extreme condition called stick-slip, wherein the drill-bit comes to a sudden stop but the top keeps in dynamic regimestoring energy - until the tip overcomes the rock resistance (Ritto, 2010; Vaziri et al., 2018). This phenomenon is present on the data and simulation of this work, modeled via dry friction. The efficiency of the CE method is compared with an Genetic Algorithm also used to solve the inverse problem.

\section{MODEL AND TEST RIG}

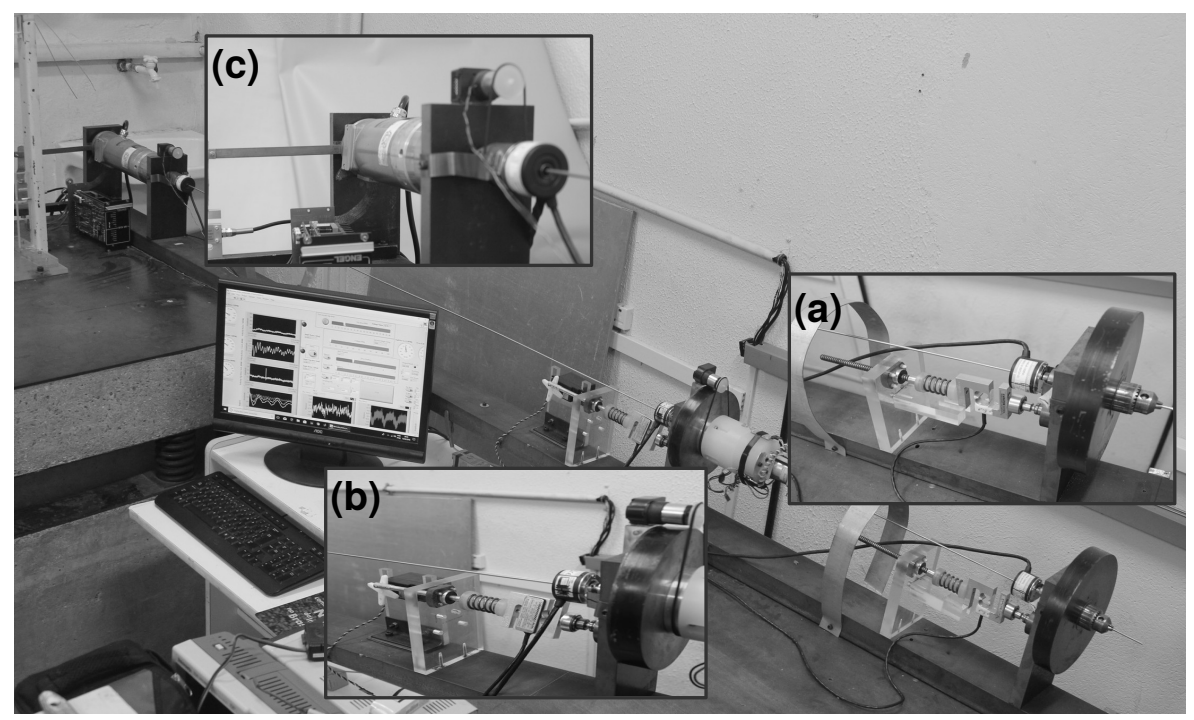

Figure 1: Test rig used for the torsional mode of a drill string. Provided by Cayres (2018).

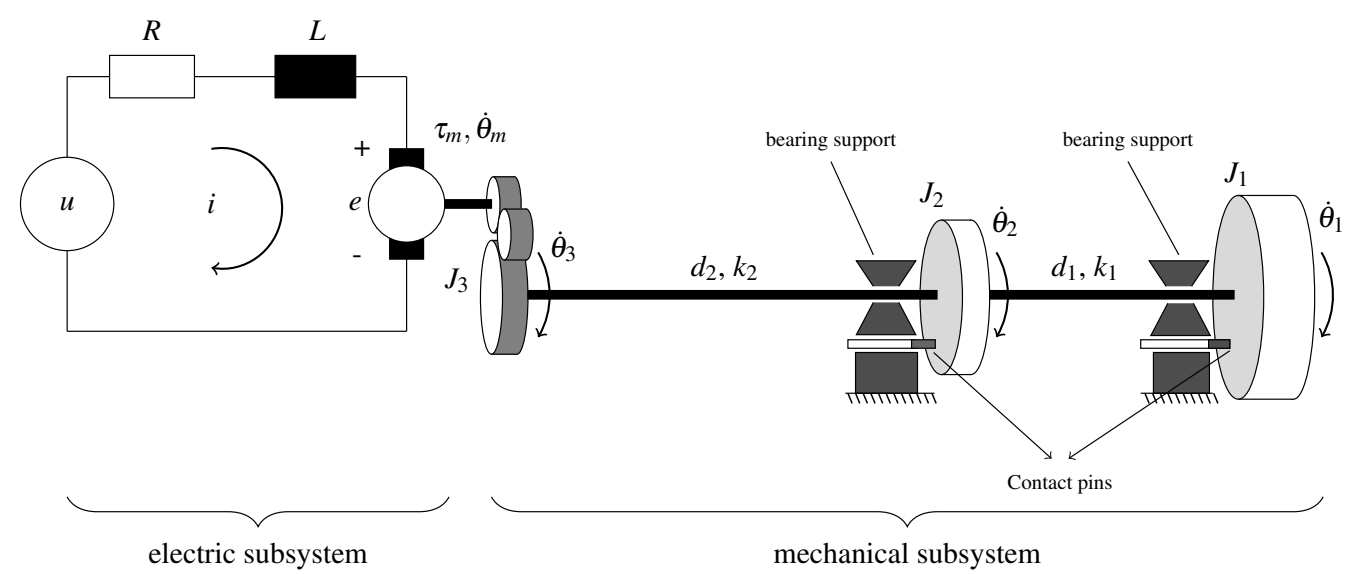

Figure 2: Schematic representation of the experimental set-up. Adapted from Pereira et al. (2018).

The data was obtained from a experimental set-up previously used by Cayres (2018) for the study of torsional vibration and stick-slip phenomenon, which can be seen in Figure 1. Figure 2 schematically summarizes the test rig, which comprises a shaft connected to three discs. The third disc (Figure 1.c) is coupled to a DC-motor that drives the dynamics. The first and second discs (Figure 1.a and 1.b, respectively) suffer dry friction via the contact pins.

The dynamics of this 2-part system is modeled as in Cayres (2018):

$$
\begin{array}{r}
J_{1} \ddot{\theta}_{1}+d_{1}\left(\dot{\theta}_{1}-\dot{\theta}_{2}\right)+k_{1}\left(\theta_{1}-\theta_{2}\right)+T_{r 1}\left(\dot{\theta}_{1}\right)=0, \\
J_{2} \ddot{\theta}_{2}+d_{1}\left(\dot{\theta}_{2}-\dot{\theta}_{1}\right)+d_{2}\left(\dot{\theta}_{2}-\dot{\theta}_{3}\right)+k_{1}\left(\theta_{2}-\theta_{1}\right)+k_{2}\left(\theta_{2}-\theta_{3}\right)+T_{r 2}\left(\dot{\theta}_{2}\right)=0, \\
d_{2}\left(\dot{\theta}_{3}-\dot{\theta}_{2}\right)+k_{2}\left(\theta_{3}-\theta_{2}\right)=\eta\left(K_{T} i-C_{m} \eta \dot{\theta}_{3}-T_{f}-J_{3} \ddot{\theta}_{3} / \eta\right), \\
L \frac{\mathrm{d} i}{\mathrm{~d} t}+R i+K_{E} \eta \dot{\theta}_{3}=u,
\end{array}
$$


where $J$ represents the moment of inertia of the discs; $d$ and $k$ mean the damping and stiffness coefficient of each string segment; $\theta, \dot{\theta}$ and $\ddot{\theta}$ are angular displacement, velocity and acceleration; $K_{T}$ is torque constant, $C_{m}$ speed regulation constant, $T_{f}$ friction torque on motor, $K_{E}$ means voltage constant (back- $E M F$ constant), $L$ inductance, $R$ resistance, $i$ is the current and the transmission factor is denoted $\eta$. The subscripts signify, as seen in Figure 2, 3 for the motor, 2 for Disc 2 (middle) and 1 for Disc 1 (end). The forcing voltage is denoted by $u$, which is modulated by a PI-controller (making the system integro-differential and non-autonomous), explicitly

$$
u=k_{p}\left(\omega_{r e f}-\dot{\theta}_{3}\right)+k_{i} \int_{0}^{t}\left(\omega_{r e f}-\dot{\theta}_{3}\right) \mathrm{d} \tau .
$$

where $\omega_{r e f}=30 \mathrm{RPM}$ is the excitation frequency, $k_{p}$ and $k_{i}$ are the controller parameters. The resistive torques applied by the contact pins provide the dry friction that enables observation of stick-slip. $T_{r 1}$ is modeled as the following (Cayres et al., 2019), with a chosen regularization of its discontinuity:

$$
T_{r 1}\left(\dot{\theta}_{1}\right)= \begin{cases}N_{1} r_{1} \mu_{s} \dot{\theta}_{1} / \omega_{s} & \text { for } \quad\left|\dot{\theta}_{1}\right|<\omega_{s}, \\ N_{1} r_{1}\left(\mu_{k}+\left(\mu_{s}-\mu_{k}\right) e^{-v_{b}\left|\dot{\theta}_{1}\right|}\right) \operatorname{sign}\left(\dot{\theta}_{1}\right) & \text { for }\left|\dot{\theta}_{1}\right| \geq \omega_{s},\end{cases}
$$

where $N_{1}$ is the normal force applied by the pin on Disc 1, $r_{1}$ is the distance of pin to the center of Disc 1 and the threshold $\omega_{s}$ is set $10^{-3} \mathrm{rad} / \mathrm{s}$. The torque on Disc 2 can be active controlled, but for the calibration presented here, $T_{r 2}=0$. The state vector of the system of Eqs. (1) consist in the relative angular displacements, angular velocities and current. Values for the prescribed parameters and experimentally identified ones (blue) can be seen in Table 1.

Table 1: Parameters of the experimental set-up of the drill string torsional dynamics. In blue, values of the parameters experimentally identified in Cayres (2018).

\begin{tabular}{clcl}
\hline Parameter & Value & Parameter & Value \\
$J_{1}$ & $0.0288 \mathrm{~kg} \mathrm{~m}^{2}$ & $L$ & $8.437 \times 10^{-4} \mathrm{H}$ \\
$J_{2}$ & $0.0149 \mathrm{~kg} \mathrm{~m}^{2}$ & $R$ & $0.33 \Omega$ \\
$J_{3}$ & $0.0237 \mathrm{~kg} \mathrm{~m}^{2}$ & $K_{T}$ & $0.126 \mathrm{~N} \mathrm{~m} / \mathrm{A}$ \\
$k_{1}$ & $1.1175 \mathrm{Nm} / \mathrm{rad}$ & $K_{E}$ & $0.0602 \mathrm{~V} \mathrm{~s} / \mathrm{rad}$ \\
$k_{2}$ & $0.3659 \mathrm{Nm} / \mathrm{rad}$ & $T_{f}$ & $0.1196 \mathrm{Nm}$ \\
$d_{1}$ & $0.0202 \mathrm{Nms} / \mathrm{rad}$ & $C_{m}$ & $1.784 \times 10^{-4} \mathrm{Nms} / \mathrm{rad}$ \\
$d_{2}$ & $0.0167 \mathrm{Nms} / \mathrm{rad}$ & $k_{p}$ & 2.800 \\
$\mu_{s}$ & 0.4700 & $k_{i}$ & 3.500 \\
$\mu_{k}$ & 0.3000 & $r_{1}$ & $0.047 \mathrm{~m}$ \\
$N_{1}$ & $16.3 \mathrm{~N}$ & $\eta$ & 8 \\
\hline
\end{tabular}

\section{FORWARD AND INVERSE PROBLEM}

The quantities of interest (QoI) in this work are the angular velocity of Disc 1 and 2. Time series for $\dot{\theta}_{1}$ and $\dot{\theta}_{2}$ are discretized in the time instants corresponding to the experimentally measured values. The forward problem consists in taking the input parameters $\mathbf{x}=\left\{k_{1}, k_{2}, d_{1}, d_{2}, \mu_{s}, \mu_{k}\right\}$ and obtaining $\mathbf{y}=\left\{\dot{\theta}_{1}, \dot{\theta}_{2}\right\}$ through numerical integration. The remaining parameters of the system are kept constant.

The inverse problem aims at finding the vector $\mathbf{x}$ that matches the real and simulated responses. A misfit function $J$ is set such that its maximization provides this, in the form of

$$
J(\mathbf{x})=\left|\rho_{1}(\mathbf{x})\right|+\left|\rho_{2}(\mathbf{x})\right|-\alpha\left\|\mathbf{x}-\mathbf{x}_{\mathbf{r}}\right\|,
$$

where $\rho_{i}$, the Pearson correlation coefficient, signifies a perfect fit at 1 , and is calculated through the expression

$$
\rho_{i}(\mathbf{x})=\operatorname{cov}\left(Y_{i}(\mathbf{x}), Y_{i}^{\text {exp }}\right) /\left(\sigma_{Y_{i}} \sigma_{Y_{i}^{e x p}}\right)
$$

with $Y_{i}$ relating to the discretized time series of $\dot{\theta}_{i} ; \sigma$ and cov are the usual standard deviation and covariance. The last term in Eq. (4) is a classical Tikhonov regularization (Engl et al., 2005) to establish a well-posed 
problem (existence, uniqueness and regularity of solution) around the background information $\mathbf{x}_{\mathbf{r}}$ : the values of the previous identified parameters (blue) in Table 1. The regularization constant $\alpha$ was chosen heuristically, via multiple trials, taking the stability of the solution provided by the methods into account.

\section{CROSS-ENTROPY METHOD}

The main idea of the cross-entropy method consists in recasting the optimization of the misfit function as a problem of estimating the probability of a rare-event. By treating the input vector as a random variable, $\mathbf{X}$, the misfit function $J(\mathbf{X})$ rarely achieves its maximum if only one exists, i.e., $J(\mathbf{X}) \geq \gamma$ is a rare event for a given level $\gamma \approx \gamma^{*}=\max J(\mathbf{x})$. A technique known as importance sampling can estimate this probability at a faster rate than a crude Monte Carlo strategy if an optimal sampling distribution is found first (D. P. Kroese, 2011). The computation of this sampling density, in turn, is related to the minimization of cross-entropy between candidate sampling densities and the (unknown) optimal one, leading to the stochastic estimator (de Boer et al., 2005)

$$
\mathbf{v}^{*}=\underset{\mathbf{v}}{\arg \max } \frac{1}{N} \sum_{i=1}^{N} I_{\left\{J\left(\mathbf{X}_{\mathbf{i}} \geq\right) \gamma\right\}} \ln f\left(\mathbf{X}_{\mathbf{i}} ; \mathbf{v}\right),
$$

where $I_{\Theta}$ is the indicator function for a given event $\Theta, N$ is number of samples, $\mathbf{v}$ is the hyperparameter of the probability density function $(\mathrm{PDF})$ and $\mathbf{v}^{*}$ is the hyperparameter of the best possible sampling PDF for this assumed family of densities.

Algorithm 1 summarizes the multi-layered procedure based on these concepts. At each step, the current PDF is used to sample parameters. Then $J(\mathbf{x})$ is evaluated and a elite group is selected, comprised by the parameters that provide its highest values. The PDF is updated via Eq. (6) using only the elite group. This is repeated until the density is concentrated around the optimal parameters.

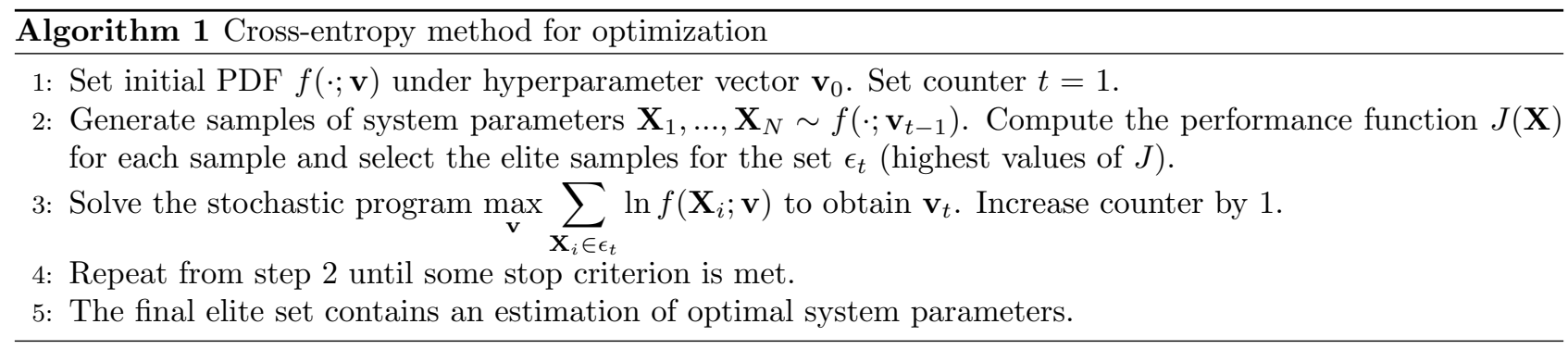

Usually, a smoothing updating rule is employed when calculating $\mathbf{v}_{t}$, and has relevant impact on convergence. A common stopping criteria, known as normal updating, is to pause the algorithm when the sum of the standard deviations is smaller than some tolerance. Both notions were used for the results of following section.

The algorithm progressively concentrates the distribution of each iteration around the unknown value $\mathbf{x}^{*}=$ $\arg \max J(\mathbf{x})$. In fact, the method is based on minimizing the Kullback-Leibler divergence between sampling PDFs and the Dirac delta distribution centered at the optimal parameter value $\mathbf{x}^{*}$ (D. P. Kroese, 2011), which "approximates" these densities at each step.

The interesting features of the CE method is that it behaves like a global search algorithm that boasts theoretical assurance to find the global optimum, if only one global optimum exists (Margolin, 2005). It also attains very easy implementation, with analytical formulas for Eq. (6); specifically, if normal distributions are used, the estimator is equivalent to the maximum likelihood estimators for mean and standard deviation (Rubinstein, 1999). Besides, it has in general very few, intuitive and defined control parameters, namely, sample size, elite set size, tolerance and smoothing parameters, which is not a common feature for other meta-heuristic procedures.

The results ahead for the context of drill string dynamics show how the efficiency of the method is comparable to a common metaheuristic strategy.

\section{RESULTS}

The inverse problem proposed by the misfit function in Eq. (4) was solved using the cross-entropy method, via Algorithm 1, for three cases, tackling an increasing number of parameters. Case A uses $\mathbf{x}=\left\{k_{1}, k_{2}\right\}$, Case $\mathrm{B}, \mathbf{x}=\left\{k_{1}, k_{2}, d_{1}, d_{2}\right\}$, and Case $\mathrm{C}, \mathbf{x}=\left\{k_{1}, k_{2}, d_{1}, d_{2}, \mu_{s}, \mu_{k}\right\}$. The upper and lower bounds considered for each parameter were $0.1-10 \mathrm{Nm} / \mathrm{rad}$ for the stiffness, $0.01-1 \mathrm{Nm} \mathrm{s} / \mathrm{rad}$ for the damping and $0.01-1$ for the friction coefficients. Figures 3 to 5 present the fitting of $\dot{\theta}_{1}$ and $\dot{\theta}_{2}$ for each Case. 

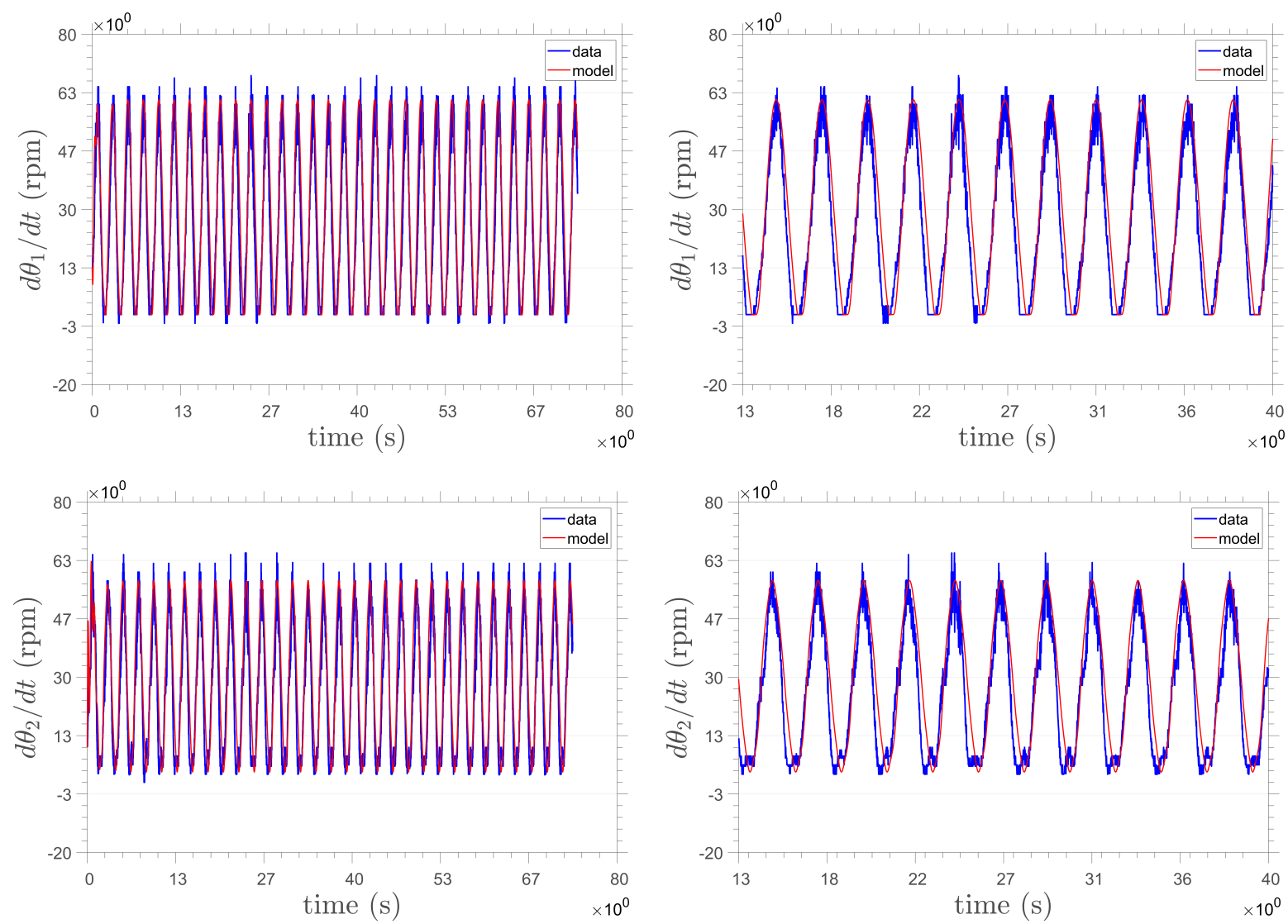

Figure 3: Calibration of angular velocities in Case $\mathrm{A}\left(\mathrm{x}=\left\{k_{1}, k_{2}\right\}\right)$. Zoomed time series on the right.
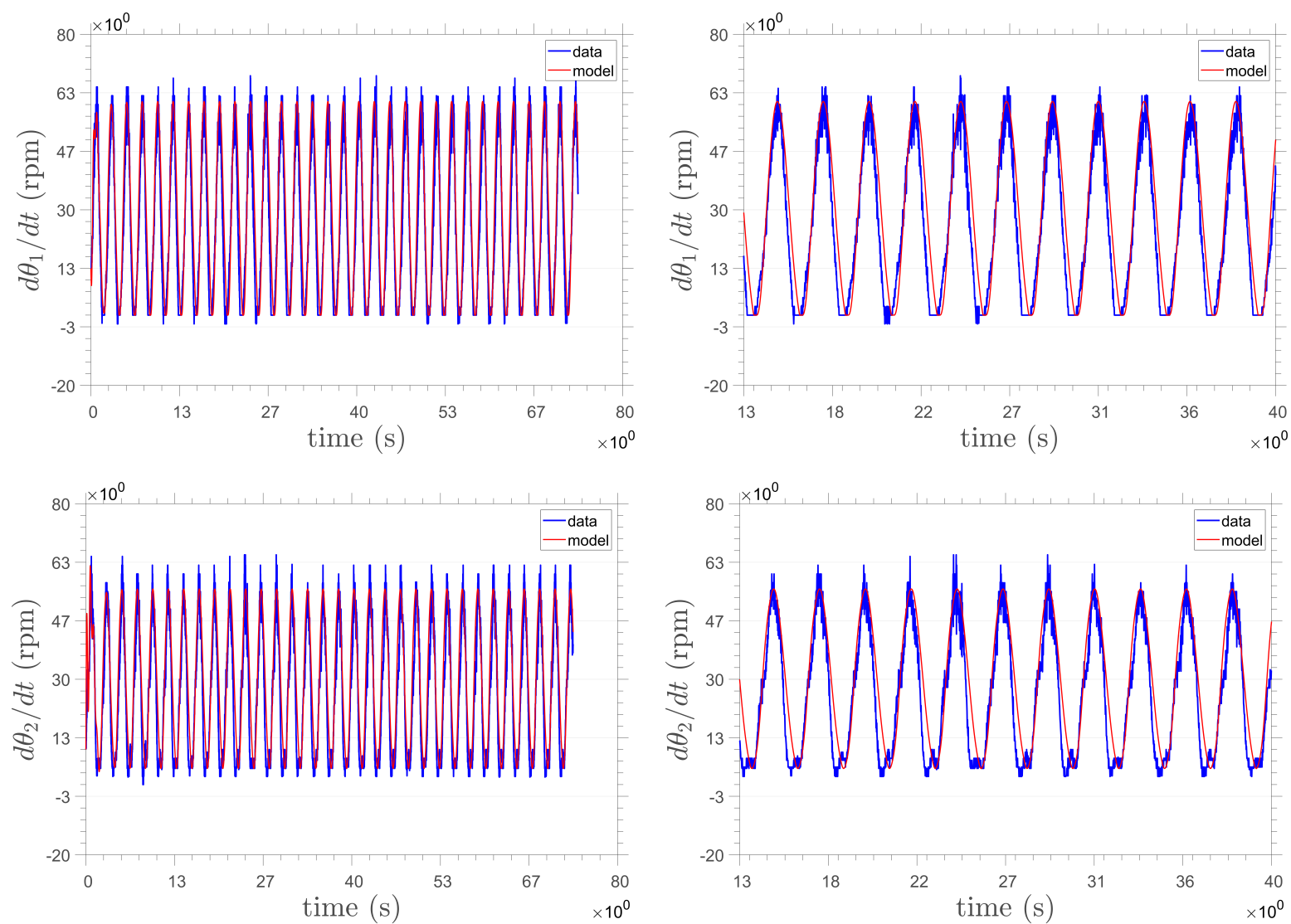

Figure 4: Calibration of angular velocities in Case $\mathrm{B}\left(\mathbf{x}=\left\{k_{1}, k_{2}, d_{1}, d_{2}\right\}\right)$. Zoomed time series on the right. 

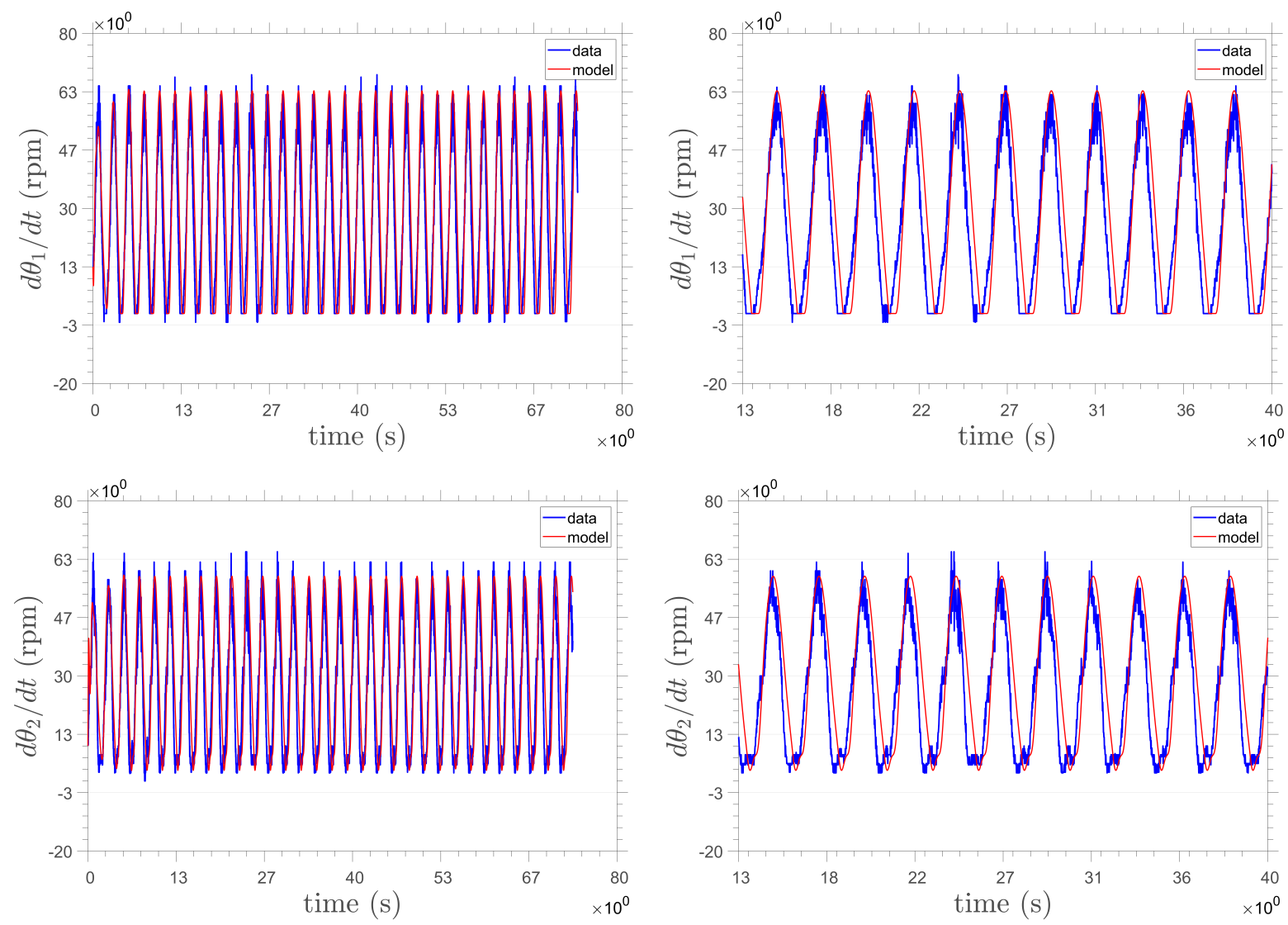

Figure 5: Calibration of angular velocities in Case $\mathrm{C}\left(\mathbf{x}=\left\{k_{1}, k_{2}, d_{1}, d_{2}, \mu_{s}, \mu_{k}\right\}\right)$. Zoomed time series on the right.

The figures clearly show an adequate solution in terms of calibrating $\dot{\theta}_{1}$ and $\dot{\theta}_{2}$, matching the overall frequency and amplitude in all cases. The lowest Pearson coefficient among all six fits was 0.9532 , thus depicting a nearperfect match. Notably, when allowing more parameters to be changed, the amplitude of the oscillation is better matched, and Case $\mathrm{C}$ even tries to replicate the high frequency variations in $\dot{\theta}_{2}$, which seem to be absent in the model responses of Case $\mathrm{A}$ and $\mathrm{B}$. This initial result reveals, as expected, that the model is capable of reproducing characteristics of the associated event, and showcase the potential for the CE method as a calibration tool for the complex torsional dynamics of drill strings, since the experimental time series is in general followed by the simulated one. This procedure can be used to fine-tune values firstly obtained empirically, to enhance a simulation. Table 2 compares the resulting values from the inverse problem with the prescribed ones found via experimental measures, and attests how the global search finds parameters close, in general, to the empirically estimated.

Table 2: Calibrated parameters for each Case of the inverse problem using the CE method. The blue values are the experimentally identified ones. Func.Eval. states how many times the forward problem was solved to reach the optimum.

\begin{tabular}{lcccc}
\hline Parameter & Experimental & Case A & Case B & Case C \\
$k_{1}(\mathrm{Nm} / \mathrm{rad})$ & 1.1175 & 1.5185 & 1.2533 & 1.4434 \\
$k_{2}(\mathrm{Nm} / \mathrm{rad})$ & 0.3659 & 0.3580 & 0.3667 & 0.3698 \\
$d_{1}(\mathrm{Nm} / \mathrm{rad})$ & 0.0202 & 0.0202 & 0.0224 & 0.0612 \\
$d_{2}(\mathrm{Nms} / \mathrm{rad})$ & 0.0167 & 0.0167 & 0.0194 & 0.0312 \\
$\mu_{s}$ & 0.4700 & 0.4700 & 0.4700 & 0.7015 \\
$\mu_{k}$ & 0.3000 & 0.3000 & 0.3000 & 0.3511 \\
Func.Eval & -- & 700 & 730 & 1,125 \\
\hline
\end{tabular}

In comparison, $k_{1}$ seems to be the hardest parameter to match the experimental values, becoming significant distant for Case C. This may be due to this input being not so sensible as the others, requiring greater changes 
in value, or may indicate the necessity of reevaluating his experimental estimate or physical modeling, since a sole parameter appeared problematic in numerical result. The fine-tuning aspect is remarkable when taking into account the validation presented in Cayres (2018), which notifies that model simulations had a significant frequency mismatch with the data during the dynamics, only to match it again later in time. While this tendency can be briefly observed in the time series shown here, such difference was greatly minimized after numerically calibrating the values. More important, it seems the frequency is maintained but the oscillation phase is significant to keep the data and model unfitted. Additionally, for a result related to the general behavior, and considering the stiff property of this system, the model appears to handle well the proposed QoIs.

An efficiency comparison with the standard Genetic Algorithm (GA) from MATLAB is presented in Table 3. The same stopping criteria was set for GA. The population size was chosen after multiple trials, seeking optimal convergence in average.

Table 3: Comparison of number of function evaluations required for CE method and GA (300 individuals per generations).

\begin{tabular}{cccc} 
Case & A & B & C \\
\hline CE & 700 & 730 & 1,125 \\
\hline GA & 3,300 & 4,200 & 3,000 \\
\hline
\end{tabular}

The CE method appears to be significantly faster as a meta-heuristic procedure in its simplest form, gaining in thousands of required function evaluations. This suggests that further modifications of the method, increasing its efficiency and making its convergence more robust, may be fruitful to tackle more complex problems.

\section{CONCLUDING REMARKS}

Identification of stiffness, damping and friction parameters for a drill string experimental set-up simulating torsional dynamics was presented, showing good fit of the results even in the presence of slip-stick phenomenon. The proposed values are realistically plausible when compared with experimental identification from a previous work. The solution of this inverse problem for a (stiff) non-autonomous dynamical system, in presence of friction effects and noisy data, suggests the utility of the cross method for this context. The comparison with Genetic Algorithm also showcases how the cross-entropy method is a comparable numerical tool even in its simplistic form. In future works, the authors intend to extend the parameter search for the motor constants using the current response, and implement variations of the cross-entropy strategy to evaluate the applicability of the method.

\section{ACKNOWLEDGEMENTS}

The authors acknowledge the support given to this research by the funding agencies Conselho Nacional de Desenvolvimento Científico e Tecnológico (CNPq), Coordenação de Aperfeiçoamento de Pessoal de Nível Superior - Brasil (CAPES) - Finance Code 001 and Carlos Chagas Filho Research Foundation of Rio de Janeiro State (FAPERJ) under grants E-26/010.002.178/2015 and E-26/010.000.805/2018.

\section{REFERENCES}

Aguiar, R.R., 2010. Experimental investigation and numerical analysis of the vibro-impact phenomenon. Ph.D. thesis, Pontifícia Universidade Católica do Rio de Janeiro, Brazil.

Cayres, B.C., 2018. Nonlinear dynamic analysis of dry friction-induced torsional vibration in a drill-string experimental set-up. Ph.D. thesis, Pontifícia Universidade Católica do Rio de Janeiro, Brazil.

Cayres, B.C., Fonseca, C.A., Sampaio, G. and Weber, H.I., 2019. "Analysis of a drill-string experimental set-up with dry friction-induced torsional vibration". In Mechanisms and Machine Science, Springer International Publishing, Vol. 62, pp. 456-469. Doi:10.1007/978-3-319-99270-9_33.

Cunha Jr, A., 2015. Modeling and Uncertainty Quantification in the Nonlinear Stochastic Dynamics of a Horizontal Dsrillstrings. Ph.D. thesis, Pontifícia Universidade Católica do Rio de Janeiro, Brazil.

Cunha Jr, A., Soize, C. and Sampaio, R., 2015. "Computational modeling of the nonlinear stochastic dynamics of horizontal drillstrings". Computational Mechanics, Vol. 56, p. 849-878.

D. P. Kroese, T. Taimre, Z.I.B., 2011. Handbook of Monte Carlo Methods. John Wiley \& Sons, 1 st edition.

Dantas, E., 2019. "A cross-entropy strategy for parameters identification problems". Monograph. Universidade 
do Estado do Rio de Janeiro.

Dantas, E., Jr, A.C. and Silva, T., 2019. "A numerical procedure based on cross-entropy method for stiffness identification". In Proceedings of the International Conference on Structural Engineering Dynamics ICEDyn 2019. https://hal.archives-ouvertes.fr/hal-02054683.

de Boer, P., Kroese, D., Mannor, S. and Rubinstein, R., 2005. "A tutorial on the cross-entropy method". Annals of Operations Research, Vol. 134, No. 1, p. 19-67. doi:10.1007/s10479-005-5724-z.

Engl, H., Hank, M. and Neubauer, A., 2005. Regularization of Inverse Problems. Kluwer Academic Publishers, 1st edition.

Eshragh, A., Filar, J.A. and Haythorpe, M., 2009. "A hybrid simulation-optimization algorithm for the hamiltonian cycle problem". Annals of Operations Research. doi:10.1007/s10479-009-0565-9.

Liao, M., Liu, Y., Chávez, J.P., Chong, A.S.E. and Wiercigroch, M., 2018. "Dynamics of vibro-impact drilling with linear and nonlinear rock models". International Journal of Mechanical Sciences, Vol. 146-147, pp. 200-210.

Margolin, L., 2005. "On the convergence of the cross-entropy method". Annals of Operations Research, Vol. 134, No. 1, pp. 201-214. doi:10.1007/s10479-005-5731-0.

Pereira, L.D., Cayres, B. and Weber, H.I., 2018. "Numerical application of a stick-slip control and experimental analysis using a test rig". MATEC Web of Conferences, Vol. 148, No. 16009.

Real, F.F., Batou, A., Ritto, T.G. and Desceliers, C., 2019. "Stochastic modeling for hysteretic bit-rock interaction of a drill string under torsional vibrations". Journal of Vibration and Control. doi:10.1177/ 1077546319828245.

Real, F.F., Lobo, D.M., Ritto, T.G. and Pinto, F.A.N.C., 2018. "Experimental analysis of stick-slip in drilling dynamics in a laboratory test-rig". Journal of Petroleum Science and Engineering, Vol. 170, pp. 755-762. doi:10.1016/j.petrol.2018.07.008.

Ritto, T.G., 2010. Numerical Analysis of the Nonlinear Dynamics of a Drill-string with Uncertainty Modeling. Ph.D. thesis, Pontifícia Universidade Católica do Rio de Janeiro, Brazil.

Rubinstein, R., 1999. "The cross-entropy method for combinatorial and continuous optimization". Methodology And Computing In Applied Probability, Vol. 1, pp. 127-190. doi:10.1023/A:1010091220143.

Rubinstein, R., 1997. "Optimization of computer simulation models with rare events". European Journal of Operational Research, Vol. 99, No. 1, pp. 89-112. doi:10.1016/S0377-2217(96)00385-2.

Rubinstein, R. and Kroese, D., eds., 2004. The Cross-Entropy Method: A Unified Approach to Combinatorial Optimization, Monte-Carlo Simulation and Machine Learning. Springer, 1st edition.

Rubinstein, R., Porotsky, S. and Kroese, D., 2006. "The cross-entropy method for continuous multi-extremal optimization". Methodology and Computing in Applied Probability, Vol. 8, No. 3, p. 383-407. doi:10.1007/ s11009-006-9753-0.

Spanos, P., Chevallier, A., Politis, N. and Payne, M., 2003. "Oil and gas well drilling: A vibrations perspective". The Shock and Vibration Digest, Vol. 35, pp. 85-103. doi:10.1177/0583102403035002564.

Vaziri, V., Kapitaniak, M. and Wiercigroch, M., 2018. "Suppression of drill-string stick-slip vibration by sliding mode control: Numerical and experimental studies". European Journal of Applied Mathematics, Vol. 29, No. 5, pp. 805-825. doi:10.1017/S0956792518000232.

Wiercigroch, M., Kapitaniak, M., Vaziri, V. and Nandakumar, K., 2018. "Complex dynamics of drill-strings: Theory and experiments". MATEC Web of Conferences. The 14th International Conference on Vibration Engineering and Technology of Machinery (VETOMAC XIV), Vol. 211, No. 01002. doi:10.1051/matecconf/ 201821101002

\section{RESPONSIBILITY NOTICE}

The authors are the only responsible for the printed material included in this paper. 